\title{
Clinico-Seroepidemiology and Molecular Characterization of Brucellosis in Animals
}

\author{
Kirit B. Patel*, H.C. Chauhan, B.K. Patel, S.S. Patel, M.D. Shrimali, J.K. Kala, A.N. Modi, \\ Rajgor Manish, M.A. Patel, A.C. Patel, M.G. Patel and B.S. Chandel
}

Department of Animal Biotechnology and Microbiology, College of Veterinary Science and Animal Husbandry Sardarkrushinagar Dantiwada Agricultural University, Sardarkrushinagar385 506, Gujarat, India

*Corresponding author

\begin{tabular}{|c|c|}
\hline & A B S T R A C T \\
\hline & \multirow{6}{*}{$\begin{array}{l}\text { The present study reports clinico seroepidemiological observations of Brucellosis } \\
\text { including molecular epidemiology. During the period of study a total of } 6410 \text { sera were } \\
\text { collected, which included cattle ( } 2723 \text { ), buffaloes ( } 894 \text { ), sheep (1072), goats (1281), camel } \\
\text { (438) and equine( } 02 \text { ). These sera were screened for the presence of Brucella antibodies by } \\
\text { RBPT and i-ELISA. The overall seroprevalence detected in animals were } 12.85 \% \text { ( } 824 \text { out } \\
\text { of } 6410 \text { ) and } 11.87 \% \text { ( } 761 \text { out of } 6410 \text { ) by RBPT and i-ELISA, respectively. Clinical } \\
\text { conditions wise seroprevalence was recorded in heifer } 4.65 \% \text { and } 6.97 \% \text {, clinically healthy } \\
\text { animals } 7.23 \% \text { and } 6.56 \% \text {, animals with the history of abortion } 25.58 \% \text { and } 29.09 \% \text {, } \\
\text { hygroma } 13.33 \% \text { and } 11.66 \% \text {, pregnant } 5.45 \% \text { and } 4.21 \% \text {, non-pregnant } 7.81 \% \text { and } \\
6.17 \% \text {, status unknown } 7.74 \% \text { and } 6.16 \% \text {, still birth } 16.00 \% \text { and } 20.00 \% \text {, retention of } \\
\text { placenta } 14.22 \% \text { and } 11.71 \% \text {, repeat breeding } 19.70 \% \text { and } 14.35 \% \text {, orchitis } 34.34 \% \text { and } \\
18.18 \% \text { respectively by RBPT and i-ELISA. Out of } 744 \text { milk samples of aborted animal, } \\
152 \text { milk samples found positive for Brucella antibody by MRT. A total } 1110 \text { clinical } \\
\text { samples were processed by genus specific PCR using B4/B5 (223bp) primer. Of these } 15 \\
\text { samples positive for Brucella organism. Out of } 15 \text { genus specific PCR positive samples, } \\
12 \text { samples positive by B. abortus }+ \text { IS711 ( } 498 \text { bp) species specific PCR and } 3 \text { samples } \\
\text { positive by B. melitensis omp31 (723bp) species specific PCR. All the } 15 \text { samples also } \\
\text { confirmed positive by Bruce ladder PCR. }\end{array}$} \\
\hline & \\
\hline $\begin{array}{l}\text { Brucellosis, } \\
\text { Seroprevalance, } \\
\text { PCR, B. abortus, } \\
\text { B. melitensis, } \\
\text { Molecular } \\
\text { detection. }\end{array}$ & \\
\hline Article Info & \\
\hline $\begin{array}{l}\text { Accepted: } \\
\text { 02 March } 2017 \\
\text { Available Online: } \\
\text { 10 April } 2017\end{array}$ & \\
\hline & \\
\hline
\end{tabular}

\section{Introduction}

Brucellosis is caused by various species of the genus Brucella, which is the second most widely spread zoonosis worldwide (Dawood, 2008). Brucella can affect almost all domestic species and cross transmission can occur between cattle, sheep, goat, camel and other species (Ghanem et al., 2009). Brucellosis is characterized by abortion and birth of nonvisible offspring in females, orchitis and epididymitis in males (Radostits et al., 2007)
(Fig. 1) It may also cause chronic inflammation of joints, tendon sheath and synovial bursa especially at the carpus (Abbas and Agab, 2002). There are many factors that can affect the prevalence of brucellosis in various species of livestock. Prevalence may vary according to climatic conditions, geography, species, sex, age and diagnostic tests used (Gul and Khan, 2007). Proper treatment and prevention of disease requires 
prompt and accurate diagnosis. Molecular diagnostics provides excellent platform for accurate and prompt diagnosis of diseases while maintaining safety of the personnel (Sola et al., 2014) and detection of Brucella organisms in various clinical, blood and serum samples of livestock and human employing PCR, Real time PCR, speciation by species specific PCR and Bruce ladder multiplex PCR. The disease has been reported from various species in different parts of India. Therefore, present study was planned for overall seroprevalence and molecular epidemiological characteristics of Brucellosis in animals.

\section{Materials and Methods}

\section{Seroprevalance}

A total 6410 Serum samples Cattle (2723), Buffaloes (894), Sheep (1072), Goats (1281), Camels (438), and equine (02) were collected from various district of Gujarat for detection of Brucella antibodies.

\section{Rose Bengal Plate Test (RBPT)}

One drop $(30 \mu \mathrm{l})$ of known Brucella antigen (IAHVB, Hebbal, Bangalore) was taken on a glass slide by micropipette then add equal amount of suspected serum samples and mixed thoroughly. The result was read immediately. Definite clumping/agglutination was considered as positive reaction, whereas no clumping/agglutination was considered as negative.

\section{Indirect ELISA}

Indirect ELISA kit was procured from National Institute of Veterinary Epidemiology and Disease Informatics (NIVEDI) Bangalore, and used as per manufacturer's protocols. The kit detects the antibodies against Brucella lipopolysaccharide (LPS) in serum samples of B. aborus and B. melitensis.

\section{Milk Ring Test (MRT) / Aborts Bang ring test (ABR)}

A total of 744 milk samples were collected in sterial vial after thorough mixing $3 \mathrm{ml}$ milk samples were taken in a test tube. Add 3 drops of $\mathrm{ABR}$ antigen and gently mixed (ABR-antigen prepared by IAHVB, Hebbal, Bangalore). The tubes were incubated at $37^{\circ} \mathrm{C}$ for one hour. Then keep at room temperature for $30 \mathrm{~min}$. Blue colored ring of cream layer at the top and absence of color in milk layer is considered as a positive. If whole milk retains blue is considered as a negative.

\section{Molecular detection of Brucella}

A total of 1110 various clinical samples were collected from cattle, buffaloes, sheep, goats, camel and equine for molecular characterization of Brucella.

\section{DNA extraction}

The genomic DNA from clinical samples was extracted using DNeasy Blood and Tissue Kit (Qiagen, USA) following manufacturers protocols.

\section{Detection of Brucella using Genus-Specific, Species-specific and Bruce-ladder PCR}

These samples processed for genus PCR, species specific PCR and Bruce-ladder PCR for confirmation using primer pair (Table 1) and conditions of thermal cycling for different primer pairs in PCR (Table 2) and PCR reaction mixture was prepared as per table 3 .

PCR amplified product was checked by running samples over $2.0 \%$ agarose gel.

\section{Results and Discussion}

\section{Seroprevalence}

Out of 6410 sera sample, overall seroprevalence recorded in animals was 
$12.85 \%$ (824 out of 6410$)$ and $11.87 \%$ (761 out of 6410) by RBPT (Fig. 2) and i-ELISA (Fig. 3) respectively (Table 4). Aulakh et al., (2008) reported over all seroprevalence of $18.26 \%$ in cattle and buffaloes from Punjab by ELISA. Gumber et al., (2004) reported over all seroprevalence of $22.5 \%$ positive in cattle and buffaloes in Punjab, by RBPT. Panchasara et al., (2015) reported overall seroprevalence was $10.66 \%, 10.29 \%$ and $9.38 \%$ by RBPT, STAT and i-ELISA, respectively in North Gujarat. However, in contrast to the present study higher rate of overall seroprevalence $28.00 \%$ was reported by Ahmed et al., (2010) by RBPT. Kushwaha et al., (2015) also reported very high seroprevalence rate of $33.85 \%, 32.61 \%$ and $30.90 \%$ by ELISA, RBPT and STAT, respectively in Pakistan. Clinical symptoms wise seroprevalence was $4.65 \%$ to $34.34 \%$ by RBPT and $4.21 \%$ to $29.09 \%$ by i-ELISA (Table 5).

Table.1 List of primers

\begin{tabular}{|c|c|c|c|}
\hline \multicolumn{4}{|c|}{ Genus specific primers: } \\
\hline $\begin{array}{c}\text { Primer } \\
\text { Forward/ } \\
\text { Reverse } \\
\end{array}$ & $\begin{array}{l}\text { Sequence } \\
\left(5^{\prime}-3^{\prime}\right)\end{array}$ & \begin{tabular}{|c} 
Product \\
size \\
(bp)
\end{tabular} & Reference \\
\hline B4 (F) & TGG CTC GGT TGC CAA TAT CAA & \multirow[t]{2}{*}{$223 \mathrm{bp}$} & \multirow{2}{*}{$\begin{array}{l}\text { Bailey et al., } \\
\text { (1992) }\end{array}$} \\
\hline $\mathrm{B} 5(\mathrm{R})$ & CGC GCT TGC CTT TCA GGT CTG & & \\
\hline \multicolumn{4}{|c|}{ " Species specific primers (B.abortus): } \\
\hline IS711(F) & "GAC GAA CGG AAT TTT TCC AAT CCC & \multirow{2}{*}{498 bp } & \multirow{2}{*}{$\begin{array}{l}\text { Bricker and } \\
\text { Halling, (1994). }\end{array}$} \\
\hline IS711(R) & TGC CGA TCA CTT AAG GGC CTT CAT & & \\
\hline \multicolumn{4}{|c|}{ Species specific primers (B.melitensis): } \\
\hline Omp31(F) & TGACAGACTTTTTCGCCGAA & \multirow{2}{*}{$723 \mathrm{bp}$} & \multirow{2}{*}{$\begin{array}{l}\text { Vizcaino et al } \\
\text { (1996) }\end{array}$} \\
\hline Omp31(R) & TATGGATTGCAGCACCGC & & \\
\hline \multicolumn{4}{|c|}{ Bruce Ladder Primers: } \\
\hline BMEI0998 (F) & ATCCTATTGCCCCGATAAGG & \multirow{2}{*}{$1682 \mathrm{bp}$} & \multirow{16}{*}{$\begin{array}{l}\text { Lopez-Goniet } \\
\text { al., (2008) }\end{array}$} \\
\hline BMEI0997(R) & GCTTCGCATTTTCACTGTAGC & & \\
\hline BMEI0535(F) & GCGCATTCTTCGGTTATGAA & \multirow{2}{*}{ 450bp } & \\
\hline BMEI0536(R) & CGCAGGGGAAAACAGCTATAA & & \\
\hline BMEII0843(F) & \begin{tabular}{|l|} 
TTTACACAGGCAATCCAGCA \\
\end{tabular} & \multirow{2}{*}{$1071 \mathrm{bp}$} & \\
\hline BMEII0844(R) & GCGTCCAGTTGTTGTTGATG & & \\
\hline BMEII436(F) & ACGCAGACGACCTTCGGTAT & \multirow{2}{*}{ 794bp } & \\
\hline BMEII435(R) & TTTATCCATCGCCCTGTCAC & & \\
\hline BMEII0428(F) & GCCGCTATTATGTGGACTGG & \multirow{2}{*}{$587 \mathrm{bp}$} & \\
\hline BMEII0428(R) & AATGACTTCACGGTCGTTCG & & \\
\hline BR0953(F) & GGAACACTACGCCACCTTGT & \multirow{2}{*}{$272 \mathrm{bp}$} & \\
\hline BR0953(R) & GATGGAGCAAACGCTGAAG & & \\
\hline BMEI0752(F) & CAGGCAAACCCTCAGAAGC & \multirow{2}{*}{$1218 \mathrm{bp}$} & \\
\hline BMEI0752(R) & GATGTGGTAACGCACACCAA & & \\
\hline BMEII0987(F) & CGCAGACAGTGACCATCAAA & \multirow[b]{2}{*}{$152 \mathrm{bp}$} & \\
\hline BMEII987(R) & GTATTCAGCCCCCGTTACCT & & \\
\hline
\end{tabular}


Table.2 Steps and conditions of thermal cycling for different primer pairs in PCR

\begin{tabular}{|c|c|c|c|c|c|c|}
\hline \multirow{2}{*}{$\begin{array}{c}\text { Primers } \\
\text { (Forward } \\
\text { and } \\
\text { Reverse) }\end{array}$} & \multicolumn{5}{|c|}{ Cycling conditions } & \multirow[b]{2}{*}{ Cooling } \\
\hline & $\begin{array}{c}\text { Initial } \\
\text { denaturation }\end{array}$ & Denaturation & Annealing & Extension & $\begin{array}{c}\text { Final } \\
\text { extension }\end{array}$ & \\
\hline \multirow{2}{*}{$\begin{array}{l}\text { B4 (F) } \\
\text { B5 (R) }\end{array}$} & $\begin{array}{l}93^{\circ} \mathrm{C} \\
5 \mathrm{~min}\end{array}$ & $\begin{array}{l}90^{\circ} \mathrm{C} \\
1 \mathrm{~min}\end{array}$ & $\begin{array}{c}64^{\circ} \mathrm{C} \\
30 \mathrm{sec}\end{array}$ & $\begin{array}{l}72^{\circ} \mathrm{C} \\
1 \mathrm{~min}\end{array}$ & $\begin{array}{c}72^{\circ} \mathrm{C} \\
10 \mathrm{~min}\end{array}$ & \multirow{8}{*}{$4{ }^{\circ} \mathrm{C}$} \\
\hline & 1 cycle & $\mathbf{R e p}_{\mathbf{P}}$ & ted for $35 \mathrm{cs}$ & & 1 cycle & \\
\hline \multirow{2}{*}{$\begin{array}{l}\text { IS711(F) } \\
\text { IS711(R) } \\
\text { (B. abortus) } \\
\end{array}$} & $\begin{array}{l}95^{\circ} \mathrm{C} \\
2 \mathrm{~min} .\end{array}$ & $\begin{array}{c}95^{\circ} \mathrm{C} \\
1.15 \mathrm{~min} .\end{array}$ & $\begin{array}{l}55.5^{\circ} \mathrm{C} \\
2 \mathrm{~min} .\end{array}$ & $\begin{array}{l}72^{\circ} \mathrm{C} \\
2 \mathrm{~min} .\end{array}$ & $\begin{array}{l}72^{\circ} \mathrm{C} \\
2 \mathrm{~min} .\end{array}$ & \\
\hline & 1 cycle & \multicolumn{3}{|c|}{ Repeated for 35 cycles } & 1 cycle & \\
\hline \multirow{2}{*}{$\begin{array}{l}\text { Omp31(F) } \\
\text { Omp31(R) } \\
\text { (B. melitensis) }\end{array}$} & $\begin{array}{c}94^{\circ} \mathrm{C} \\
5 \mathrm{~min} .\end{array}$ & $\begin{array}{l}94^{\circ} \mathrm{C} \\
1 \mathrm{~min} . \\
\end{array}$ & $\begin{array}{c}58^{\circ} \mathrm{C} \\
1 \mathrm{~min} .\end{array}$ & $\begin{array}{l}72^{\circ} \mathrm{C} \\
2 \mathrm{~min} .\end{array}$ & $\begin{array}{c}72^{\circ} \mathrm{C} \\
10 \mathrm{~min} .\end{array}$ & \\
\hline & 1 cycle & \multicolumn{3}{|c|}{ Repeated for 35 cycles } & 1 cycle & \\
\hline \multirow{2}{*}{$\begin{array}{c}\text { Bruce } \\
\text { Ladder } \\
\text { Primers }\end{array}$} & $\begin{array}{l}95^{\circ} \mathrm{C} \\
7 \text { min }\end{array}$ & $\begin{array}{c}95^{\circ} \mathrm{C} \\
35 \text { second }\end{array}$ & $\begin{array}{c}64^{\circ} \mathrm{C} \\
45 \mathrm{sec}\end{array}$ & $\begin{array}{l}72^{\circ} \mathrm{C} \\
3 \mathrm{~min}\end{array}$ & $\begin{array}{l}72^{\circ} \mathrm{C} \\
6 \mathrm{~min}\end{array}$ & \\
\hline & 1 cycle & \multicolumn{3}{|c|}{ Repeated for 25 cycles } & 1 cycle & \\
\hline
\end{tabular}

Table.3 Various components used in PCR

\begin{tabular}{|c|c|c|c|}
\hline Components & $\begin{array}{l}\text { Genus/ Species } \\
\text { specific PCR }\end{array}$ & Components & $\begin{array}{l}\text { Bruce ladder } \\
\text { PCR }\end{array}$ \\
\hline $\begin{array}{l}\text { PCR Master Mix } \\
(2 X)\end{array}$ & $12.5 \mu 1$ & PCR Master Mix (2X) & $12.5 \mu \mathrm{l}$ \\
\hline $\begin{array}{l}\text { Forward Primer } \\
(10 \mathrm{pmol} / \mu \mathrm{l})\end{array}$ & $1 \mu l$ & \multirow{2}{*}{$\begin{array}{l}\text { Bruce-ladder eight pair primer } \\
\text { cocktail }(12.5 \mu \mathrm{M})\end{array}$} & \multirow{2}{*}{$1 \mu l$} \\
\hline $\begin{array}{l}\text { Reverse Primer } \\
(10 \mathrm{pmol} / \mu \mathrm{l})\end{array}$ & $1 \mu l$ & & \\
\hline Template DNA & $2 \mu \mathrm{l}$ & Template DNA & $2 \mu 1$ \\
\hline Nuclease free water & $8.5 \mu \mathrm{l}$ & Nuclease free water & $8.5 \mu \mathrm{l}$ \\
\hline Total & $25 \mu l$ & Total & $25 \mu \mathrm{l}$ \\
\hline
\end{tabular}

Table.4 Overall seroprevalence

\begin{tabular}{|c|c|c|c|}
\hline \multirow{2}{*}{ Species } & No. of tested & \multicolumn{2}{|c|}{ Seroprevalance } \\
\cline { 3 - 4 } & & $\begin{array}{c}\text { RBPT } \\
\text { positive }\end{array}$ & $\begin{array}{c}\text { i-ELISA } \\
\text { positive }\end{array}$ \\
\hline Cattle & 2723 & $333(12.22 \%)$ & $307(11.27 \%)$ \\
\hline Buffalo & 894 & $129(14.42 \%)$ & $116(12.97 \%)$ \\
\hline Goat & 1281 & $123(9.60 \%)$ & $108(8.43 \%)$ \\
\hline Sheep & 1072 & $170(15.85 \%)$ & $171(15.95 \%)$ \\
\hline Camel & 438 & $69(15.75 \%)$ & $59(13.47 \%)$ \\
\hline Equine & 02 & $00(0.00 \%)$ & $00(0.00 \%)$ \\
\hline Total & $\mathbf{6 4 1 0}$ & $\mathbf{8 2 4}(\mathbf{1 2 . 8 5 \%})$ & $\mathbf{7 6 1 ( 1 1 . 8 7 \% )}$ \\
\hline
\end{tabular}


Table.5 Clinical status wise seroprevalence

\begin{tabular}{|l|l|l|l|}
\hline \multirow{2}{*}{ Symptoms } & \multirow{2}{*}{ No. of tested } & Seroprevalance & \\
\cline { 3 - 4 } & & RBPT positive & i-ELISA positive \\
\hline Heifer & 172 & $08(4.65 \%)$ & $12(6.97 \%)$ \\
\hline Clinically healthy & 2087 & $151(7.23 \%)$ & $137(6.56 \%)$ \\
\hline Abortion & 110 & $284(25.58 \%)$ & $323(29.09 \%)$ \\
\hline Hygroma & 180 & $24(13.33 \%)$ & $21(11.66 \%)$ \\
\hline Pragnant & 403 & $22(5.45 \%)$ & $17(4.21 \%)$ \\
\hline Non-pragnant & 486 & $38(7.81 \%)$ & $30(6.17 \%)$ \\
\hline Status unknown & 762 & $59(7.74 \%)$ & $47(6.16 \%)$ \\
\hline Still birth & 50 & $08(16.00 \%)$ & $10(20.00 \%)$ \\
\hline Retension of Placenta & 239 & $34(14.22 \%)$ & $28(11.71 \%)$ \\
\hline Repeat breeding & 822 & $162) 19.70 \%)$ & $118(14.35 \%)$ \\
\hline Orchitis & 99 & $34(34.34 \%)$ & $18(18.18 \%)$ \\
\hline Total & $\mathbf{6 4 1 0}$ & $\mathbf{8 2 4}(\mathbf{1 2 . 8 5 \%})$ & $\mathbf{7 6 1}(\mathbf{1 1 . 8 7 \%})$ \\
\hline
\end{tabular}

Table.6 Processing of milk samples by MRT

\begin{tabular}{|c|c|c|}
\hline Species & No. of tested & $\begin{array}{c}\text { MRT } \\
\text { positive }\end{array}$ \\
\hline Cattle & 584 & $116(19.86 \%)$ \\
\hline Buffalo & 154 & $36(23.37 \%)$ \\
\hline Camel & 06 & $00(0.00 \%)$ \\
\hline Total & $\mathbf{7 4 4}$ & $\mathbf{1 5 2}(\mathbf{2 0 . 4 3 \%})$ \\
\hline
\end{tabular}

Fig.1 Clinical symptoms
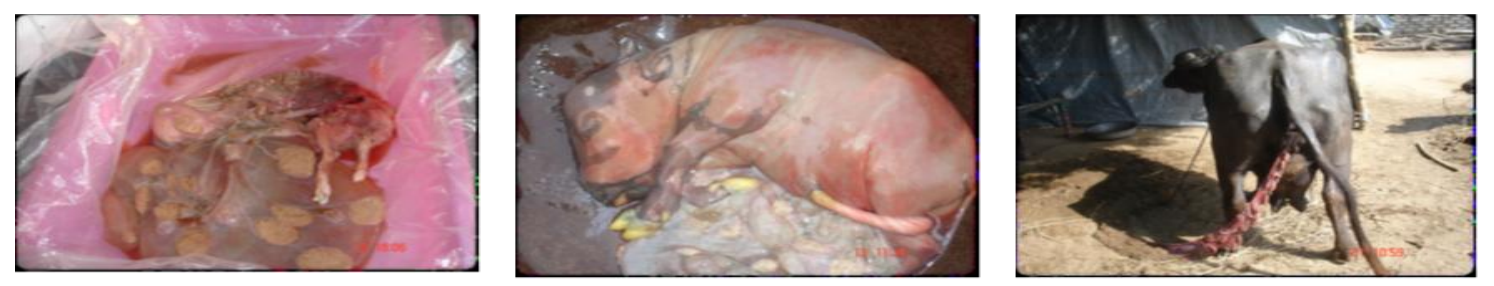

Abortion

Retension of placenta
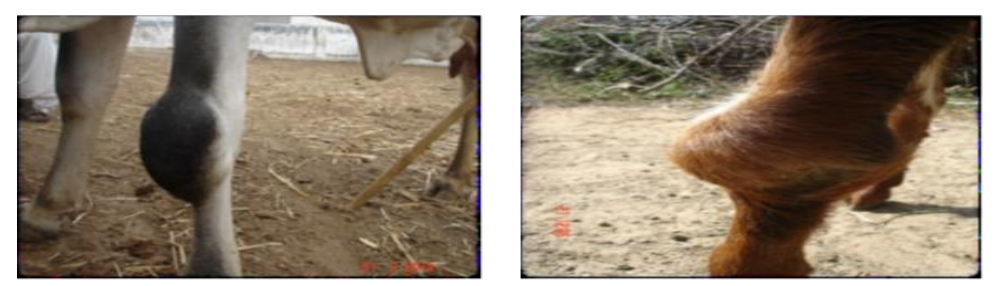

Hygroma

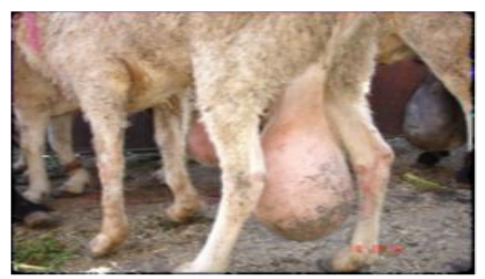

Orchitis 
Table.7 Direct detection of Brucella by PCR

\begin{tabular}{|c|c|c|c|c|c|c|c|c|}
\hline \multirow[t]{2}{*}{ Type of sample } & \multicolumn{6}{|c|}{ Species } & \multirow{2}{*}{$\begin{array}{l}\text { No.of } \\
\text { tested }\end{array}$} & \multirow{2}{*}{$\begin{array}{l}\text { Result } \\
\text { No. of sample } \\
\text { positive in } \\
\text { PCR }\end{array}$} \\
\hline & Cattle & Buffalo & Sheep & Goat & Camel & Equine & & \\
\hline Blood & 231 & 102 & 178 & 111 & 91 & 09 & 722 & 00 \\
\hline Vaginal swab & $73^{*}$ & $45^{*}$ & 37 & 31 & 03 & 02 & 191 & 02 \\
\hline $\begin{array}{l}\text { Vaginal } \\
\text { discharge }\end{array}$ & $08 * *$ & $05^{*}$ & $02^{*}$ & 00 & 00 & 00 & 15 & 04 \\
\hline Milk & 12 & 08 & 04 & 06 & 03 & 00 & 33 & 00 \\
\hline Placenta & $17 * *$ & $09 * *$ & $04^{*}$ & 01 & 00 & 00 & 31 & 05 \\
\hline Placental fluid & 05 & 02 & 00 & 00 & 00 & 00 & 07 & 00 \\
\hline Hygroma fluid & 02 & 01 & 00 & 00 & 00 & 00 & 03 & 00 \\
\hline Amniotic fluid & 00 & 02 & 00 & 00 & 00 & 00 & 02 & 00 \\
\hline Orchitis fluid & 00 & 00 & 03 & 01 & 00 & 00 & 04 & 00 \\
\hline Foetal intestine & 00 & 00 & 03 & 01 & 00 & 00 & 04 & 00 \\
\hline $\begin{array}{l}\text { Foetal intestine } \\
\text { fluid }\end{array}$ & 02 & 01 & 01 & 02 & 00 & 00 & 06 & 00 \\
\hline Foetal lung & $09^{*}$ & 04 & 02 & 06 & 00 & 00 & 21 & 01 \\
\hline Foetal liver & 09* & 04 & 02 & 06 & 00 & 00 & 21 & 01 \\
\hline $\begin{array}{l}\text { Foetalabomasal } \\
\text { content }\end{array}$ & 00 & 00 & $03^{*}$ & 02 & 00 & 00 & 05 & 01 \\
\hline $\begin{array}{l}\text { Foetal stomach } \\
\text { content }\end{array}$ & $03^{*}$ & 01 & 03 & 05 & 00 & 00 & 12 & 01 \\
\hline Foetal heart & 03 & 00 & 02 & 02 & 00 & 00 & 07 & 00 \\
\hline $\begin{array}{l}\text { Foetal heart } \\
\text { blood }\end{array}$ & 06 & 04 & 02 & 04 & 00 & 00 & 16 & 00 \\
\hline Cotyledon & 06 & 04 & 00 & 00 & 00 & 00 & 10 & 00 \\
\hline Total & 386 & 192 & 246 & 178 & 97 & 11 & 1110 & 15 Sample \\
\hline
\end{tabular}

*indicate number of positive sample in PCR

Fig.2 Rose Bengal test

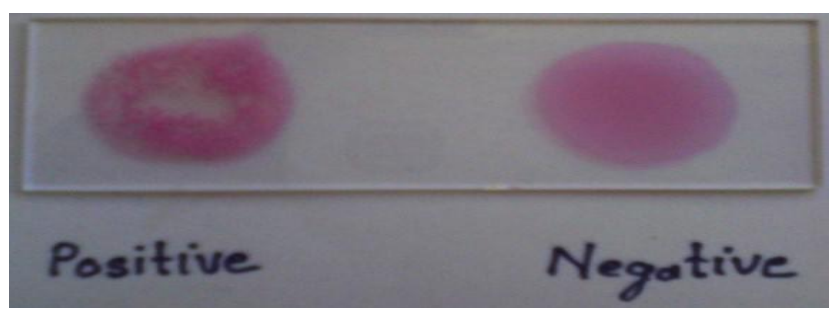


Fig.3 i-ELISA

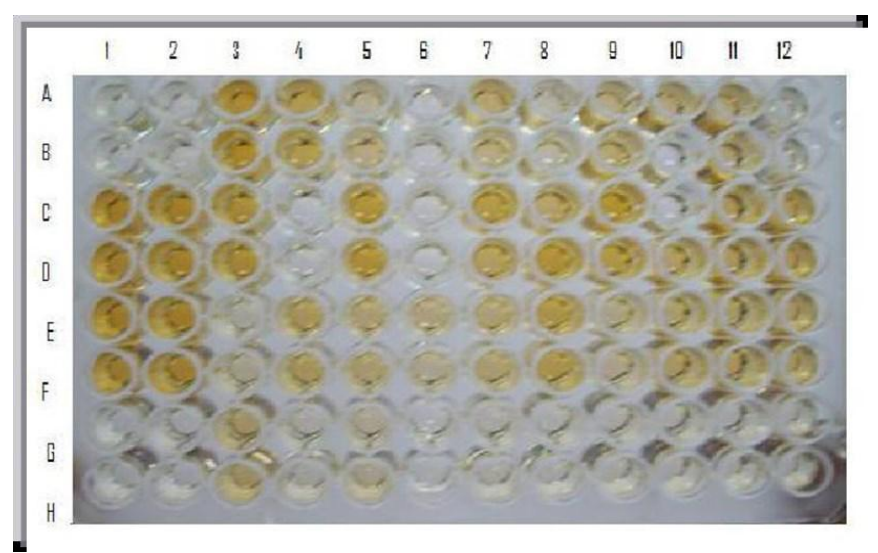

Wells A1, B1, A2, B2: Negative control; Wells C1, D1, C2, D2: Moderately positive control Wells E1, F1, E2, F2: Strong positive control; Wells A3, B3, C3, D3, C5, D5 etc. positive field sera reaction

Fig.4 Milk ring test

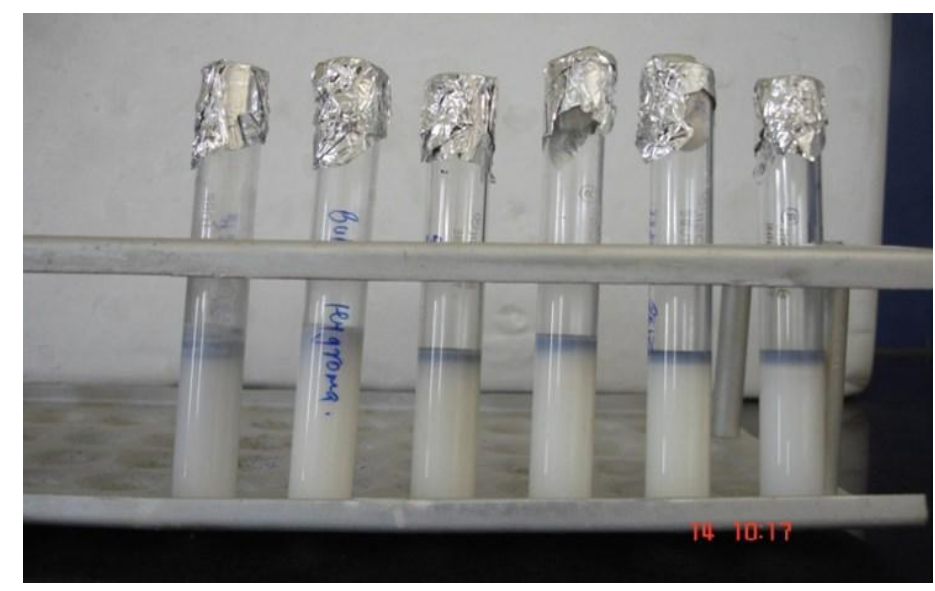

Fig.5 Genus specific PCR223bp PCR products with B4B5 primer

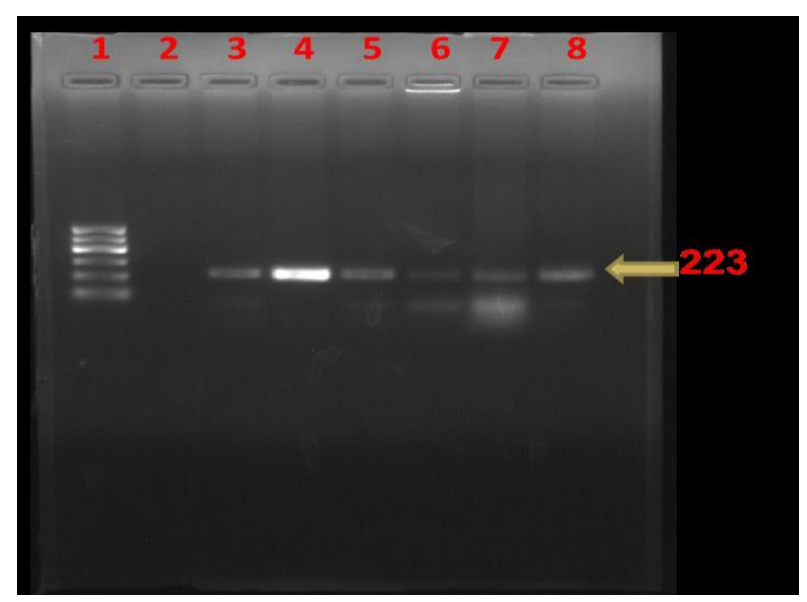

1-ladder

2-NTC

3- sample (positive)Vaginal discharge

4- sample (positive) Foetal lung

5-sample (positive)Placenta

6- sample (positive)Foetal liver

7- sample (positive)Foetal stomach content 
Fig.6 Species specific PCR495 bp PCR product with IS711 primer

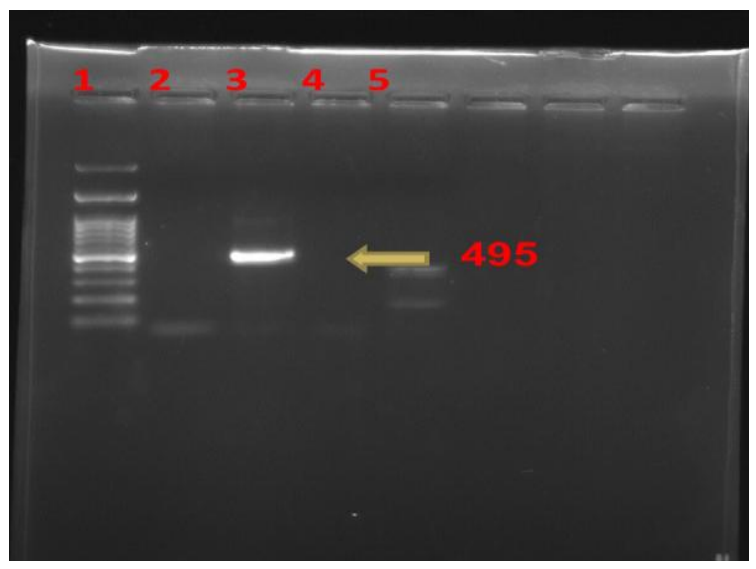

1-ladder

2-NTC

3- sample (positive)Foetal lung

4- sample (negative)

5-sample (negative)

Fig.7 Species specific PCR 723bp PCR product with omp31 primer

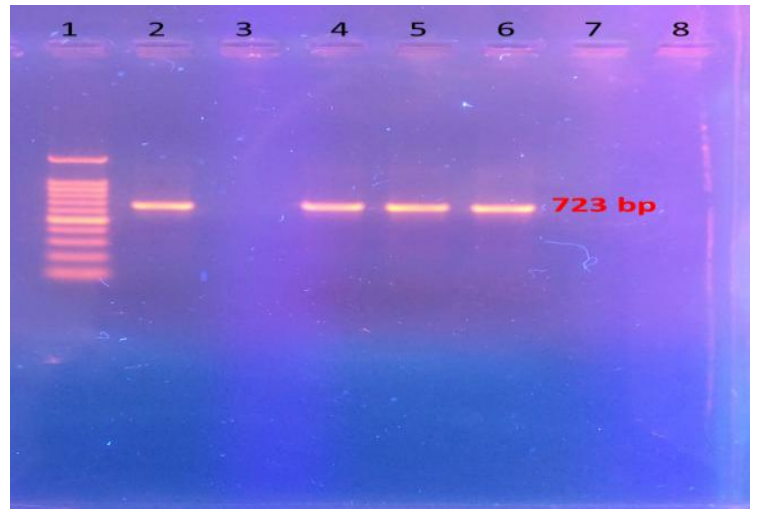

1- Ladder

2-Positive control

3- NTC

4-Sample (positive) vaginal discharge

5- Sample (positive) fetal abomasal content

6- Sample (positive) placenta

Fig.8 Bruce ladder PCR

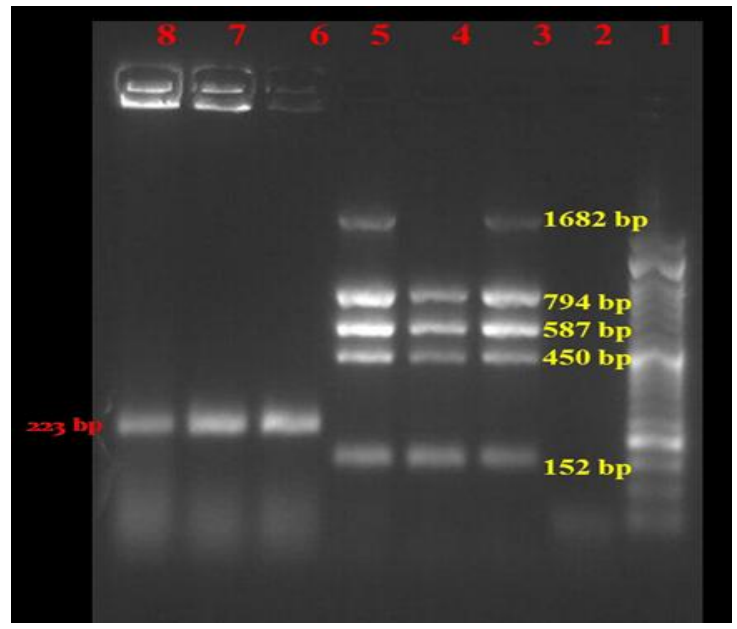

1- Ladder

2-NTC

3- Sample (positive)Placenta (Bruce ladder)

4-Sample (positive) vaginal discharge (Bruce ladder)

5- Sample (positive) vaginal swab (Bruce ladder)

6- Sample (positive) placenta (Genus Specific)

7- Sample (positive) vaginal discharge (Genus Specific)

8- Sample (positive) vaginal swab(Genus Specific)

Out of 744 milk samples, of these $152(20.43 \%)$ samples were found positive for Brucella antibodies by MRT (Fig. 4; Table 6). Present finding was in agreement with Zowghi et al., (1990) which detected $25.21 \%$ Brucella antibody from milk by MRT. However, In 
contrast to the present findings, Al-Mariri (2015) reported 9.16\%and Gulluce et al., (1996) reported $56.32 \%$ Brucella antibody from milk by MRT.

\section{Molecular detection of Brucella from clinical samples}

In PCR study targeting 16S rRNA gene, Out of 1110 clinical samples fifteen samples (Table 7) were found positive to give specific amplicon of $223 \mathrm{bp}$ region of the sequence encoding a 31 $\mathrm{kDa}$ immunogenic bcsp31 by Brucella genus specific primer pairs B4/B5 (Fig. 5). Out of 15 genus specific positive samples, 12 samples yielded an amplicon of 498bp in +IS711 primers indicate species as Brucella abortus (Fig. 6) and 3 samples yielded an amplicon of $723 \mathrm{bp}$ in omp31 primers indicate species as Brucella melitensis (Fig. 7). All 15 genus specific positive samples were also confirmed as positive by Bruce ladder PCR (Fig. 8). Similarly, Kanani (2007) and Jung et al.,(1998) detection of Brucella by using bcsp31 gene based B4/B5primer.Earlier Navarro et al., (2002), Kanani (2007) and Patel (2007) used same primer pairs for molecular detection of Brucella abortus. Patel et al., (2015) and Karthik et al., (2014) used species specific +IS711 primers for detection of Brucella abortus and they yielding 498bp band and Vizcaino et al., (1996)used species specific omp31 primers for detection of $B$. melitensis and they yielding 723bp band when electrophoresed through $2 \%$ agarose gel. Lopez Goni et al., (2008) reported Bruce-ladder was species specific and all the strains and biovars from the same Brucella species gave the same profile.

\section{Acknowledgement}

We are highly thankful to DBT, Govt. of India for financial assistance for the project.

\section{References}

Abbas, B. and Agab, H. 2002. A review of camel brucellosis. Preventive Vet. Med., 55: 47-56.
Abouzeed, Y.M., Ibrahim, A., Salem, H., Alzwam, F., Abid, S., Elfahem, A. and Elrais, A. 2010. Seroprevalence of brucellosis in animals and human populations in the western mountains region in Libya, December 2006-January 2008. Euro Surveill., 15(30): 19625.

Al-Mariri. 2015. Isolation of Brucella melitensis strains from Syrian bovine milk samples. Bulgarian J. Vet. Med., 18(1): 40.

Aulakh, H.K., Patil, P.K., Sharma, S., Kumar, H., Mahajan, V. and Sandhu, K.S. 2008. A Study on the Epidemiology of bovine brucellosis in Punjab (India) using milkELISA. Acta Veterinaria Brno., 77: 393399.

Bailey, G.G., Krahn, J.B., Drasar, B.S. and Stoker, N.G. 1992. Detection of Brucella melitensis and Brucella abortus by DNA amplification. J. Trop. Med. Hygiene, 95: 271-275.

Bricker and Halling. 1994. Differentiation of Brucella abortus bv. 1, 2 and 4 Brucella melitensis, Brucella ovis and Brucella suis bv 1 by PCR. J. Clin. Microbiol., 32: 2660-2666.

Dawood, H.A. 2008. Brucellosis in camels (Camelusdro medorius) in the south province of Jordan. Am. J. Agric. Biol. Sci., 3: 623-626.

Ghanem, Y.M., El-Khodery, S.A., Saad, A.A., Abdelkader, A.H., Heybe, A. and Musse, Y.A. 2009. Seroprevalance of camel brucellosis (Camelisdro medarus) in Somaliland. Trop. Anim. Health Prod., 41: 1779-1786.

Gul, S.T. and Khan, A. 2007. Epidemiology and Epizootology of Brucellosis: A Review. Pak. Vet. J., 27:145-151.

Gulluce, M. and Leloglu, N. 1996. Detection of Brucella abortus antibodies in cow milk of the Kars area by ELISA and MRT. Turkish J. Vet. Animal Sci., 20(4): 251255.

Gumber, S., Aradhana, A., Dhand, N.K. and Sandhu, K.S. 2004. Village level study of bovine brucellosis in Punjab (India) by 
bulk milk analysis. Indian J. Animal Sci., 74: 843-844.

Jung, S.C., Jung B.Y., Woo, S.R., Cho, D.H., Kim, J.Y., Kim, W.T., Lee, J.M., Park, Y.H. and Baek, B.K. 1998. Development of a PCR assay for the detection of Brucella spp. in bovine semen. Korean J. Vet. Res., 38: 345-352.

Kanani, A.N. 2007. Serological, cultural and molecular detection of Brucella infection in breeding bulls. $\mathrm{Ph}$. D. thesis submitted to A. A. U., Anand.

Karthik, K., Rathore, R., Thomas, P., Arun, T.R., Viswas, K.N., Agarwal, R.K., Manjunathachar, H.V. and Dhama, K. 2014. Loop-mediated isothermal amplification (LAMP) test for specific and rapid detection of Brucella abortus in cattle. Vet. Quarterly, 34(4): 174-179.

Kushwaha, N., Rajora, V.S., Mohan, A., Upadhyay, A.K. and Kumar, R. 2015. Comparison of serological tests for detection of Brucella antibodies in cattle of an organized dairy farm. Indian $J$. Animal Res., 50(1): 69-74.

Lopez-Goni, I., Garcia-Yoldi, D., Marin, C.M., Miguel, M.J., Munoz, P.M., Blasco, J.M., Jacques, I., Grayon, M., Cloeckaert, A., Ferreira, A.C., Cardoso, R., Correa de Sa, M.I., Walravens, K., Albert, D. and Garin-Bastuji, B. 2008. Evaluation of a multiplex PCR assay (Bruce-ladder) for molecular typing of all Brucella species, including the vaccine strains. J. Clin. Microbiol., 46: 3484-3487.

Navarro, E., Escribano, J., Fernandez, J. A. and Solera, J. 2002. Comparison of three different PCR methods for detection of Brucella spp. in human blood samples. FEMS Immunol. Med. Microbiol., 34:
147-151.

Panchasara, H.H., Patel, J.S. and Patel, P.R. 2015. Seroepidemiological pattern of brucellosis among buffaloes in north Gujarat. Indian J. Vet. Sci. Biotechnol., 11(1): 74-79.

Patel, B.C., Chauhan, H.C., Chandel, B.S., Dadawala, A.I. and Jain., B.K. 2015. Seroprevalence and Molecular characterization of Brucella spp. in buffalo from North Gujarat, India. Int. J. Curr. Microbiol. Appl. Sci., 4(4): 174180.

Patel, T.J. 2007. Serological, cultural and molecular detection of Brucella infection in bovines including quantification in milk by real-time PCR. An M.V. Sc. thesis submitted to A. A. U., Anand.

Radostits O.M., Gay C., Blood D.C. and Hinchclift K.W. 2007. Disease associated with helminthes parasite. In: veterinary medicine, a text book of the disease of cattle, sheep, goat, pigs and horses. 10th ed. London: Bailliere, Tindall, UK, 984988.

Sola, M.C., Da Veiga, J.E.A., De Freitas, M.R. and De Mesquita, A.J. 2014. Real-time PCR detection of Brucella spp. DNA in lesions and viscera of bovine carcasses. $J$. Microbiol. Methods, 104: 87-91.

Vizcaino, N., Cloeckaert, A., Zygmunt, M.S. and Dubray, G. 1996. Cloning, nucleotide sequence and expression of omp31 gene coding for an immunogenic major outer membrane protein. Infect. Immunity, 64(9): 3744-3751.

Zowghi, E., Ebadi, A. 1990. Mohseni, B. Isolation of Brucella organisms from the milk of seronegative cow. Rev. sci. Tech. Off. Int. Epiz., 9(4): 1175-1178.

\section{How to cite this article:}

Kirit B. Patel, H.C. Chauhan, B.K. Patel, S.S. Patel, M.D. Shrimali, J.K. Kala, A.N. Modi, Rajgor Manish, M.A. Patel, A.C. Patel, M.G. Patel and Chandel, B.S. 2017. Clinico-Seroepidemiology and Molecular Characterization of Brucellosis in Animals. Int.J.Curr.Microbiol.App.Sci. 6(4): 222-231. doi: https://doi.org/10.20546/ijcmas.2017.604.026 